\title{
Propolis derivatives inhibit the systemic inflammatory response and protect hepatic and neuronal cells in acute septic shock
}

\section{Authors}

Aida Abdelhamid Korish Maha Mohamed Arafa ${ }^{2}$

${ }^{1} \mathrm{PhD}$; Associate Professor of Physiology, Department of Physiology, Faculty of Medicine, King Saud University, Riyadh, Saudi Arabia

${ }^{2}$ KSUFSP; Assistant Professor of Pathology, Department of Pathology, Faculty of Medicine, King Saud University, Riyadh, Saudi Arabia
Submitted on: 01/09/2011 Approved on: 03/20/201

Correspondence to: Aida Abdelhamid Korish King Saud University, Faculty of Medicine Department of Physiology 2925, Riyadh 11461, Saudi Arabia iaidakorish@yahoo.com

Financial Support: The study was supported by the College of Medicine Research Center, Faculty of Medicine King Saud University, grant \#07-580.

We declare no conflict of interest.

\begin{abstract}
Background: Severe pathogenic infection triggers excessive release of cytokines as part of the massive inflammatory response associated with septic shock. Objectives: To investigate the protective effect of caffeic acid phenethye ester (CAPE) against lipopolysaccharide (LPS) induced endotoxemia, hepatic and neuronal damage and the associated systemic inflammatory response (SIR). Methods: Fifty male Wister rats were divided into: control, LPS, and CAPE+LPS groups. Plasma concentrations of various cytokines, including TNF- $\alpha$, IL-1 $\alpha$, IL-1 $\beta$, IL-6, IL-4, IL-10, and sICAM-1 were evaluated. In addition, the histopathological changes in the hepatic and neural cells were assessed. Results: The LPS group showed high inflammatory cytokines and sICAM-1 levels reflecting the presence of SIR. Hepatocyte necrosis, apoptosis, extensive hemorrhage and inflammatory cellular infiltration together with brain astrocytes swelling, early neuron injury and presence of inflammatory foci confirmed the toxic tissue damage. Use of CAPE decreased the inflammatory cytokines and increased the anti-inflammatory cytokines levels. This biochemical evidence of decreased SIR was confirmed histologically by decreased cellular infiltration in the liver and brain tissue which coincides with preserved structure and protection of the liver and brain cells from the toxic effects of LPS. Conclusion: The ability of CAPE to alleviate the SIR, hepatic and neuronal cell damage induced by LPS and galactosamine could be attributed to its ability to reverse the imbalance of the pro- and anti-inflammatory cytokines which may lead to the inhibition of adhesion molecules' expression. CAPE is a promising agent that could help in the prophylaxis and treatment of septic shock.
\end{abstract}

Keywords: lipopolysaccharides; septic shock; systemic inflammatory response syndrome; cytokines.

\section{INTRODUCTION}

Despite major advances in critical care medicine, derangement of the immune system with disturbed pro- and anti-inflammatory cytokines production and systemic inflammatory response syndrome (SIRS) remain significantly high, accounting to $40-70 \%$ of deaths in intensive care units. ${ }^{1}$ Septic shock is a whole body massive inflammatory response triggered by systemic infection leading to uncontrolled inflammatory response on the cellular and humoral defense mechanisms with excessive inflammatory cytokines production, disordered vascular control manifested as hypotension and peripheral vasodilatation refractory to intravascular volume replacement and vasopressor drugs. This leads to organ hypoperfusion with consequent multiple organ failure (MOF), and circulatory collapse. ${ }^{2}$
Lipopolysacharide (LPS) is an integral component of the outer membrane of Gram-negative bacteria and plays a major role in the pathogenesis of septic shock. ${ }^{3}$ The combination of LPS and D-galactosamine (GalN) is used to induce experimental septic shock, and is also used frequently to induce liver injury in rodents that simulate acute liver failure in clinic. ${ }^{3,4} \mathrm{D}$-galactosamine augments the LPSinduced elevation of serum tumor necrosis factor-alpha (TNF- $\alpha$ ), and sensitizes the animal to it. Metabolism of GalN by the hepatic enzymes participating in the galactose pathway leads to consumption and rapid depletion of the uridine nucleotides in the liver, and to a decrease in RNA synthesis. ${ }^{5}$ LPS induced lethality is characterized by liver failure, accompanied by severe hepatic injury through enhanced TNF- $\alpha$ production; so toxic shock and fulminate hepatitis with severe hepatic 
congestion ensue resulting in rapid death within few hours of the combined injection of LPS and GalN. ${ }^{4}$ Under the influence of endotoxins, hepatic stellate cells (HSCs) can secrete a variety of chemokines and cytokines that contribute to hepatic damage. ${ }^{6}$ Several microbes and microbial products that induce liver injury stimulate the transcription and synthesis of interleukin (IL)- $1 \beta$ which causes growth of fibroblasts, proliferation of lymphocytes, induction of adhesion molecules and stimulation ofother cytokines and inflammatory mediators production in an autocrine and paracrine way. ${ }^{7}$

The pathophysiology of septic shock is complex, involving multiple endogenous mediators as cytokines, nitric oxide (NO), and cycloxygenase 2 (COX2). ${ }^{8,9}$ Experimental and clinical evidence indicate that patients with sepsis suffer from severe oxidative stress with overproduction of reactive oxygen species (ROS) and reactive nitrogen species (RNS), which modulate cell adhesion and cause direct injury to the endothelium..$^{10}$ The first line of defense against infection is polymorphonuclear leucocytes (neutrophils and macrophages), which act as a double-sided sword; where its recruitment and activation localize, kill and clear the pathogen; ${ }^{11}$ whereas uncontrolled sequestration and activation produce a large number of powerful pro-inflammatory mediators that play a key role during the onset of sepsis through the development of extensive systemic inflammation and crucial tissue injury implicated in multi-organ damage and malfunction. ${ }^{12}$ Several previous results suggest that LPS regulates nitric oxide synthase (NOS) and prostaglandin E2 (PGE2) production from activated macrophages by controlling the activation of nuclear transcription factor kappa B (NF- $\mathrm{kB}$ ). Binding of $\mathrm{NF}-\kappa \mathrm{B}$ to its binding sites on the promoter region of the gene encoding inducible NOS (iNOS) plays an important role in LPS-induced upregulation of iNOS gene. ${ }^{8}$ This high expression of iNOS contributes to the pathogenesis of septic shock. ${ }^{13}$

Despite better understanding of the pathophysiology of sepsis and although many treatment strategies have been used for septic shock, yet the mortality rate did not improve substantially, ${ }^{2}$ because of the complex interaction of the inflammatory mediators with the contribution of multiple intracellular pathways. ${ }^{9}$ This leads researchers in this field to have as a goal the search for a therapeutic measure to attenuate excessive acute inflammatory response without compromising essential host defense mechanisms. Caffeic acid phenethyl ester (CAPE), a natural derivative of the honeybee propolis, ${ }^{14}$ is a small lipid soluble potent flavenoid with multiple biological effects that emerged in recent research as antioxidant ${ }^{15}$ and anti-inflammatory agent. ${ }^{16}$ It passes rapidly into blood after intraperitoneal (IP) injection and due to its lipophylic nature and small molecule size, it enters easily into cells by crossing cell membrane. This may explain the marked effects obtained with low doses. ${ }^{16} \mathrm{CAPE}$ is a specific and potent inhibitor of the activation of NF- $\mathrm{KB},{ }^{17}$ which predispose it to the proposed therapeutic effect for treatment of inflammatory diseases. ${ }^{18}$ Several recent studies investigated the effect of CAPE treatment in carbon tetrachloride (CCL4), cisplatin, and LPS induced toxicity. ${ }^{19-21}$ Where it was found to exert antioxidant and anti-inflammatory effects characterized by increased activity of the antioxidative enzymes, decrease ROS, and inflammatory cytokines production. This was reflected in better liver function tests and decreased signs of tissue damage and inflammatory cellular infiltration in diseased organs. But to our knowledge, the role of CAPE as a prophylactic measure against the SIRS and the hepatocellular and neuronal cell damage associated with endotoxic septic shock has not yet been investigated and needs to be studied. Therefore, the present study focused on the possible protective role of the prophylactic use of CAPE, when used two weeks before the exposure to LPS/GalN induced septic shock on hepatic and brain damage and on the associated inflammatory cytokines production.

\section{METHODS}

\section{Animals and diet}

Fifty adult (2-3 months old) male Wister rats (230-250 g) were kept in the animal house of the Faculty of Medicine, King Saud University (KSU) under standard laboratory conditions, with controlled temperature $\left(23-25^{\circ} \mathrm{C}\right)$ and $12 \mathrm{~h}$ light: dark cycles with free access to water, rodent chow $\mathrm{ad}$ libitum. The study design was approved by the College of Medicine Research Center, KSU and all the experimental procedures were done in accordance with the standard established guidelines of Laboratory Animal Care Committee of the Faculty of Medicine, KSU and Helsinki Declaration for experimental studies.

Animals were divided into three experimental groups: control sham group $(n=10)$, LPS group $(n=20)$, and CAPE+ LPS group $(n=20)$. From day 1 until day 14 of the study, CAPE+LPS group received daily IP injection of $10 \mu \mathrm{mol} / \mathrm{kg}$ CAPE (Sigma-Aldrich, St Louis, MO, USA) while the control and LPS groups were injected with saline. On day 15 of the study, endotoxic shock was induced in LPS \& CAPE+LPS groups with single IP injection of $20 \mathrm{mg} / \mathrm{kg}$ LPS (from Escherichea coli, Serotype O55:B5, Sigma-Aldrich) dissolved in PBS, and GalN (250 mg/kg). Control animals received vehicle only $(200 \mu \mathrm{L})$. After 30 minutes of LPS injection, animals in the CAPE+LPS group received a single IP injection of CAPE in the same dose given before while both LPS and control groups were injected with saline. Among rats receiving LPS, death rate was about $30 \%$ within 6 hours. Six hours after LPS injection, animals were anesthetized with pentobarbital sodium $(40 \mathrm{mg} / \mathrm{kg}$ body weight) and blood samples were obtained by cardiac puncture in EDTA tubes for the biochemical analysis. Then the animals were scarified, liver and brain were rapidly dissected, washed with cold saline and kept in $10 \%$ formalin 
solution for histological examination. Plasma was separated by blood centrifugation at $2,000 \mathrm{~g}$ for $20 \mathrm{~min}$ at $4^{\circ} \mathrm{C}$ and was stored at $-70^{\circ} \mathrm{C}$ until analyses.

\section{Laboratory studies}

Plasma concentrations of interleukins were measured with Specific Rat ELISA kits for IL-1 $\alpha$, IL-1 $\beta$, IL-4, IL-6, IL-10 (R\&D), and TNF- $\alpha$ (Biosource). Rat soluble intercellular adhesion molecule-1(sICAM-1) was measured by Quantikine ELISA kit (R\&D) according to the manufacturer instructions. The techniques were conducted using Automated Microplate Reader - model ELX800, serial number 191720, (Bio Tek Instruments Inc, Vermont, USA). The mean minimum detectable doses of the kits were $19.5 \mathrm{pg} / \mathrm{mL}$ for TNF- $\alpha, 10.0 \mathrm{pg} / \mathrm{mL}$ for IL-10, $21 \mathrm{pg} / \mathrm{mL}$ for IL-6, $5 \mathrm{pg} / \mathrm{mL}$ for IL-4, $1.24 \mathrm{pg} / \mathrm{mL}$ for IL- $1 \alpha$, $5 \mathrm{pg} / \mathrm{mL}$ for IL- $1 \beta$ and $2.0 \mathrm{pg} / \mathrm{mL}$ for sICAM- 1 .

\section{Histopathological assessment}

Control rat livers macroscopically look red in color but the livers of the LPS injected groups turned dark red to black in color and look very congested. Tissue specimens were embedded individually into paraffin blocks, then serially sectioned into 5 - $\mu \mathrm{m}$-thick samples, stained with hematoxylin-eosin, and examined under light microscopy (Olympus BX 51) and pictures were taken by Olympus camera DP 72 at $4 \mathrm{X}, 10 \mathrm{X}, 40 \mathrm{X}$ and $60 \mathrm{X}$. Brain tissue was investigated for astrocytes and neuron cell structural changes and liver samples were examined for hepatic stellate cells structural changes. In addition, the presence of hemorrhage, inflammatory cellular infiltration, nuclear swelling, cytoplasmic vacuolization, focal necrotic islets, and single cell death was also examined.

\section{Statistical analysis}

Results were analyzed using the SPSS package for statistical analysis. Comparison between all groups was done by using one way ANOVA. A post Hoc LSD was used when ANOVA give significant differences. All data are presented as mean \pm S.E.M. Results were considered significant when $\mathrm{p}<0.05$. Due to the death of some animals in both LPS and LPS+CAPE groups, the actual number included in the statistics was 12 rats in each of these groups.

\section{RESULTS}

\section{Biochemical results}

\section{Pro-inflammatory cytokines}

TNF- $\alpha$

As shown in Table 1, there was significant increase in plasma TNF- $\alpha$ levels in untreated LPS rats in comparison to the control group $(530.5 \pm 89.56 v s .7 .18 \pm 0.28 \mathrm{pg} / \mathrm{mL}$, $\mathrm{p}=0.000)$. CAPE+LPS rats showed significant decrease in their plasmatic TNF- $\alpha$ levels $(194.2 \pm 29.78 \mathrm{pg} / \mathrm{mL})$ in comparison to the untreated LPS rats $(\mathrm{p}=0.000)$.

$I L-1 \alpha$

IL-1 $\alpha$ levels increased significantly in LPS group reaching a mean value of $593.7 \pm 49.83 \mathrm{pg} / \mathrm{mL} v$ s. $6.57 \pm 0.42 \mathrm{pg} / \mathrm{mL}$ in the control group $(\mathrm{p}=0.000)$. CAPE treatment caused marked significant decrease in IL-1 1 level to $217.59 \pm 34.86 \mathrm{pg} / \mathrm{mL}$ in comparison to non-CAPE treated LPS rats $(\mathrm{p}=0.000)($ Table 1$)$.

$I L-1 \beta$

Plasma IL-1 $\beta$ increased significantly in LPS group in comparison to the control group (326.48 $\pm 78.39 v s$. $15.01 \pm 0.39 \mathrm{pg} / \mathrm{mL}, \mathrm{p}=0.000$ ). CAPE treatment for two weeks

Table 1. Plasma cytokines and soluble intercellular adhesion molecule (sICAM-1) in all studied groups

\begin{tabular}{|c|c|c|c|c|c|}
\hline Parameters & $\begin{array}{c}\text { Control } \\
n=10\end{array}$ & $\begin{array}{c}\text { LPS } \\
\mathbf{n}=12\end{array}$ & $\begin{array}{c}\text { CAPE+LPS } \\
\mathbf{n}=12\end{array}$ & $\mathbf{p}$ & $\mathbf{F}$ \\
\hline TNF- $\alpha$ & $7.18 \pm 0.28$ & $530.5 \pm 89.56^{*}$ & $194.2 \pm 29.78^{\dagger}$ & 0.000 & 25.96 \\
\hline IL-1 $\alpha$ & $6.57 \pm 0.42$ & $593.7 \pm 49.83^{*}$ & $217.59 \pm 34.86^{* \dagger}$ & 0.000 & 84.01 \\
\hline $\mathrm{IL}-1 \beta$ & $15.01 \pm 0.39$ & $326.48 \pm 78.39^{*}$ & $158.10 \pm 13.30^{*}+$ & 0.000 & 13.09 \\
\hline IL-6 & $38.33 \pm 1.62$ & $3471.66 \pm 325.20^{*}$ & $2800.83 \pm 90.26^{*}$ & 0.000 & 291.98 \\
\hline IL-4 & $29.79 \pm 1.10$ & $29.49 \pm 0.55$ & $32.15 \pm 0.93^{\dagger}$ & 0.08 & 2.70 \\
\hline IL-10 & $116.0 \pm 1.74$ & $645.82 \pm 44.68^{*}$ & $874.88 \pm 38.18^{* \dagger}$ & 0.000 & 120.76 \\
\hline sICAM-1 & $483.88 \pm 26.56$ & $2942.2 \pm 139.08^{*}$ & $2517.98 \pm 136.08^{* \dagger}$ & 0.000 & 59.34 \\
\hline
\end{tabular}

All data are presented as mean \pm S.E.M. Results were considered significant when $\mathrm{p}<0.05$, LPS; acute septic shock group receiving lipopolysacharide (LPS) $20 \mathrm{mg} / \mathrm{kg}$ (from Escherichea coli ,serotype O55:B5, Sigma-Aldrich) dissolved in PBS, and D-galactosamine (GalN) $250 \mathrm{mg} / \mathrm{kg}$, CAPE+LPS; septic shock group treated with caffeic acid phenethylester (CAPE) $10 \mu \mathrm{mol} / \mathrm{kg}$. $\mathrm{p}<0.05$ versus control group;

${ }^{\dagger} \mathrm{p}<0.05$ versus LPS group. 
significantly decreased plasma IL- $1 \beta$ levels in the CAPE+LPS group in comparison to the untreated LPS group with a mean value of $158.10 \pm 13.3 \mathrm{pg} / \mathrm{mL}(\mathrm{p}=0.015)$ (Table 1$)$.

\section{IL-6}

Untreated LPS rats showed tremendous increase in their plasma levels of IL-6 with a mean value of $3471.66 \pm 325.20 \mathrm{pg} / \mathrm{mL}$ that was significantly higher than the control value $(38.33 \pm 1.62 \mathrm{pg} / \mathrm{mL}, \mathrm{p}=0.000)$. CAPE treatment decreased IL-6 level in the CAPE+LPS group to $2800.83 \pm 90.26 \mathrm{pg} / \mathrm{mL}$ that was significantly lower than that of the non-CAPE treated LPS group $(\mathrm{p}=0.004)$ (Table 1).

\section{Anti-inflammatory cytokines}

\section{IL-4}

Although IL-4 levels did not change significantly in LPS group in comparison to the control group $(29.49 \pm 0.55$ vs. $29.79 \pm 1.10 \mathrm{pg} / \mathrm{mL}, \mathrm{p}=0.08)$. CAPE treatment was effective in causing a significant increase in IL-4 levels $(32.15 \pm 0.93 \mathrm{pg} / \mathrm{mL})$ in CAPE+LPS rats in comparison to LPS group ( $p=0.039$ ), but this increase in IL-4 in CAPE+LPS group showed no significant difference from the control group $(\mathrm{p}=0.07)($ Table 1$)$.

\section{IL-10}

IL-10 increased significantly in LPS group $(645.82 \pm 44.68 \mathrm{pg} / \mathrm{mL})$ in comparison to the control rats $(116.0 \pm 1.74 \mathrm{pg} / \mathrm{mL}, \mathrm{p}=0.000)$. CAPE treatment caused further and significant increase in IL-10 level in CAPE+LPS group $(874.88 \pm 38.18 \mathrm{pg} / \mathrm{mL}, \mathrm{p}=0.000)$ in comparison to LPS group (Table 1).
sICAM-1

Soluble ICAM-1 levels increased significantly in LPS group reaching $2942.2 \pm 139.08 \mathrm{pg} / \mathrm{mL}$ versus control value $(483.88 \pm 26.56 \mathrm{pg} / \mathrm{mL})$. CAPE treated group showed significantly lower levels of sICAM-1 $(2517.98 \pm 136.08 \mathrm{pg} / \mathrm{mL})$ than LPS group $(\mathrm{p}=0.007)($ Table 1$)$.

\section{Histopathology}

A crucial aspect during sepsis is acute organ failure. In comparison to control group (Figure 1, left), LPS-induced endotoxemia and organ damage was confirmed in LPS group (Figure 1, middle) by the presence of histopathological picture of the liver injury characterized by severe hemorrhagic necrosis, destruction of the architecture of hepatic lobules, massive infiltration by inflammatory cells like neutrophils and macrophages in portal area and in the hepatic tissue causing hepatocellular necrosis. Apoptosis of the hepatocytes and the presence of acidophilic bodies (counsel man bodies) and extensive multifocal hemorrhage in liver tissues were also found. Rats receiving pretreatment with CAPE showed significantly reduced liver damage showing itself with intact hepatic lobules and normal hepatocytes shape with a definite margin between the nucleus and cytoplasm, marked reduction in the hemorrhage, the inflammation and the necrosis (Figure 1, right). Regarding the changes in the brain, in comparison to the histological picture of the control rats (Figure 2, left), untreated LPS endotoxemia group showed signs of early neuron injury, focal inflammatory cellular infiltration, and mild cytoplasmic swelling of the astrocytes (Figure 2, middle),
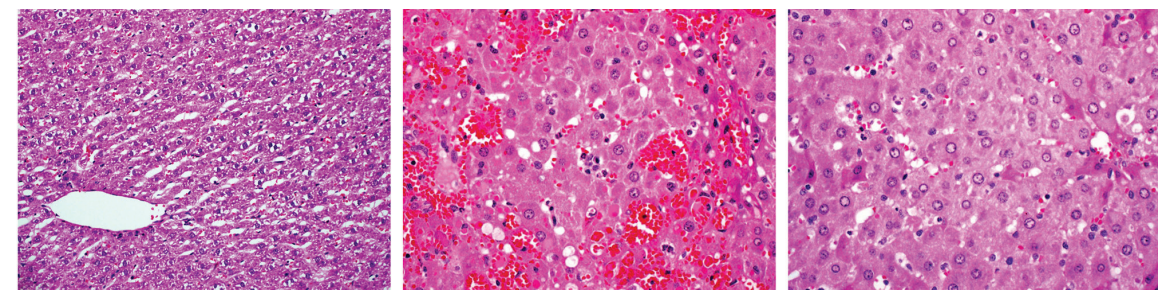

Figure 1: 40X image of liver of control (left), untreated acute septic shock (middle) showing destruction of the hepatic lobules with marked infiltration by neutrophilic granulocytes, obstruction of sinusoids, endothelial cell damage and indistinct boundaries between the nucleus and cytoplasm, so much hemorrhage, hepatocyte necrosis. Acute septic shock group receiving caffeic acid phenethylester (right) showing normal hepatic lobules, hepatocytes are normal in shape, clearly visible margins between hepatocytes nucleus and cytoplasm, no apparent hemorrhage nor inflammatory cellular infiltration.
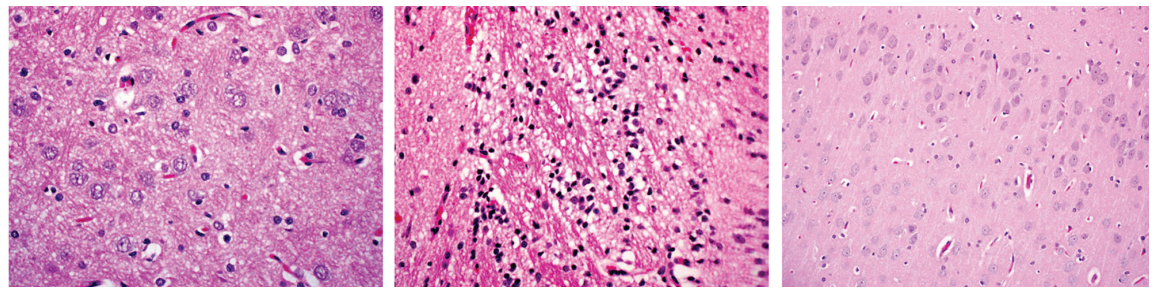

Figure 2: 40X image of brain tissue of control group (left), untreated acute septic shock group (middle) showing lymphocytic infiltration and swollen astrocytes and early neuron injury, while brain of the caffeic acid phenethyle ester treated septic shock group (right) normal astrocytes along the tracts, normal neurons structure, and absent inflammatory cellular infiltration. 
which may be a manifestation of brain edema. CAPE treatment preserved the histological picture of the brain with absent inflammatory cellular infiltration and intracellular edema (Figure 2, right).The brain tissue of the CAPE treated rats looks almost like the control brain.

\section{DISCUSSION}

Septic shock is a complex pathophysiological process where a plethora of biologically active molecules play a role in its development. ${ }^{9}$ Therefore, therapeutic strategies directed against a specific biomarker were not successful in controlling the problem, and the mortality rate remains unacceptably high. This raises the need for a therapeutic agent with multiple physiological and pharmacological activities. Pathogenic bacteria and their products, such as LPS, trigger the activation of NF- $\mathrm{KB}$, which plays a central role in the induction of networks between cytokines and the inflammatory mediators, leading to the pathophysiology of septic shock. ${ }^{21}$ During inflammatory stimulation, translocation of NF- $\mathrm{KB}$ from the cytosol into the nuclei of cells induces the expression of a large number of genes involved in inflammation; including those encoding cytokines (IL-1, IL-6 and TNF- $\alpha$ ), adhesion molecules, acute phase proteins and enzymes such as NOS. ${ }^{22}$ Pro-inflammatory cytokines and inflammatory mediators stimulate increased production of Cox-2 in macrophages and endothelial cells which contribute to the edema and vasodilatation at the inflammation site. ${ }^{8}$ Activation of NF- $\mathrm{kB}$ and its consequences occurs in all organs causing organ damage that eventually lead to organ failure with increased morbidity and mortality of patients. ${ }^{22}$ This makes NF- $\mathrm{kB}$ an attractive therapeutic target for the pharmacological control of endotoxemia. Among the multiple beneficial effects reported for the natural honeybee propolis, CAPE - the active derivative of propolis - has an inhibitory effect on NF$\kappa \mathrm{B}$ activation with anti-inflammatory ${ }^{17}$ and antioxidant activities. ${ }^{15}$ This encourages us to investigate the potential protective effect of CAPE in the complex problem of septic shock with specific emphasis on its effect on the pro-inflammatory and anti-inflammatory cytokines production and the pathological changes induced by LPS/GalN in the liver and brain tissues during the process of endotoxemia.

Findings of the present study reveal marked increase in the pro-inflammatory cytokines IL- $1 \alpha$, IL- $1 \beta$, IL- 6 and TNF- $\alpha$ levels in animals with LPS endotoxemia. This coincides with death of $30 \%$ of the animals within six hours of LPS injection. This could be due to the circulatory collapse and organ hypoperfusion and damage associated with the acute endotoxemia. ${ }^{2}$ Locally, the liver was very much congested and dark in color (with histological picture of hepatic necrosis, intra-hepatic hemorrhage and marked hepatocellular infiltration by neutrophils and macrophages). This could be explained by the ability of LPS and D-GalN to trigger activation of the immune cells as PMNL, monocytes and Kupffer cells causing infiltration of the damaged liver with these cells. Activated macrophages send fluxes of cytokines, chemokines, superoxide $[\mathrm{O}(2)-], \mathrm{NO}$, hypochloric acid (HOCL), and hydrogen peroxide $\left(\mathrm{H}_{2} \mathrm{O}_{2}\right)$ into the ECF that induce oxidative stress and cause more cellular injury. ${ }^{8,23}$ Released pro-inflammatory cytokines subsequently induce inflammatory changes and release vasoconstrictive mediators as endothlin-1 and thromboxane causing necrosis of endothelial cells with subsequent fibrin deposition in the sinusoids. ${ }^{24}$ In contrast to healthy organs endotoxin perfused liver cannot adapt to reduced portal blood flow by a compensatory increase in arterial perfusion, consequently, decreased blood flow impairs the function of the hepatocytes and Kupffer cells. ${ }^{25}$

Increased plasma levels of the inflammatory cytokines IL- $1 \alpha$, IL- $1 \beta$, IL- 6 , and TNF- $\alpha$ detected in the LPS group in the present study, reveals a picture of systemic inflammatory reaction. In addition, IL-10 which is known to have both inflammatory and anti-inflammatory action ${ }^{26}$ was also found to be elevated in the LPS group of animals in the current study. This elevation of IL-10 could be part of the SIR picture, but it may act as compensatory mechanism to inflammation, through its anti-inflammatory action. CAPE treatment was found to be associated with significant increase in IL-10 and IL-4 levels in the treated group. This may be explained by the potential anti-inflammatory effect of CAPE which decreases the release of the inflammatory cytokines from the inflammatory cells and in the same time stimulates increased production of anti-inflammatory cytokines like IL-10 and IL-4. Inflammatory cellular infiltration of the hepatic tissue was reported to be associated with Fas/FasL pathway activation with subsequent activation of caspase- 3 and 8 leading to apoptosis of the liver cells. ${ }^{27}$ Adhesion molecules like ICAM-1 regulate the interaction between T-lymphocytes and hepatocytes and this relation is deregulated in harmful inflammatory processes. ${ }^{28}$ Increased sICAM-1 levels were verified in the plasma of the non-CAPE treated LPS group in the current study. The elevation of sICAM-1 was reported to trigger the extravasations of neutrophils in the liver parenchyma producing more cytotoxic damage to hepatocytes. ${ }^{28}$ This together with accumulated intracellular metabolic end products functionally overloads the residual liver, impairs DNA synthesis and may inhibit liver regeneration. ${ }^{29}$ LPS induced hepatotoxicity ruins liver function manifested by elevated liver transaminases and bilirubin levels and abnormal blood coagulation tests. ${ }^{21}$ Use of CAPE treatment in the CAPE+LPS group in the present study was able to decrease the infiltration of the liver cells by the inflammatory cells like neutrophils and monocytes, which is suggested to strengthen the defense mechanisms against ROS into the liver cells and attenuate the excessive inflammatory injury induced by IL- $1 \alpha$, IL- $1 \beta$, TNF- $\alpha$ and IL- 6 , as demonstrated by decreased plasma levels of these cytokines in the treated group. The ability of CAPE to prevent the formation of malondial- 
dehyde (MDA) and ROS was reported in ischemic injury ${ }^{16}$ at a concentration of $10 \mu \mathrm{mol} / \mathrm{kg}$ (similar to that used in our study), and was suggested to underlie the preventive effect of CAPE against these injuries. CAPE treatment also reduced the inflammation and lung damage induced by LPS in rats. ${ }^{29}$ ROS promote activities of pro-inflammatory redox sensitive nuclear factors, including NF- $\mathrm{KB}$ which is detected in various cell types and has been determined to have a critical function in immune and inflammatory responses. ${ }^{30,31}$ Activation of NF- $\mathrm{KB}$ is associated with increased expression of matrix metaloprotinase-9 (MMP-9), IL- $1 \beta$, and IL- 8 that in turn enhances NF- $\kappa B$ activation in different cell systems. ${ }^{32}$ CAPE, being a specific inhibitor of the activation of NF- $\kappa \mathrm{B},{ }^{17}$ blocked IL- $1 \beta$-induced $\mathrm{NF}-\kappa \mathrm{B}$ promoter activation and was recently reported to be a potent inhibitor of NF- $\kappa B$ activity in several pathologies that were attributed to increased oxidative stress and neutrophil accumulation..$^{33}$ These reported actions of CAPE give us an explanation to the beneficial effects obtained with CAPE treatment in the present study. Our findings clearly demonstrate that CAPE treatment in a dose of $10 \mu \mathrm{mol} / \mathrm{kg}$ for two weeks before the exposure to LPS and a single dose after LPS-induced endotoxemia markedly protect against LPS-induced hepatocellular and neural damage in treated rats together with decreased inflammatory cytokines production. This may be through its anti-inflammatory ${ }^{16}$ and free radical scavenging properties ${ }^{15}$ that may underlie the decreased pro-inflammatory cytokines levels (IL1- $\alpha$, IL1- $\beta$, IL- 6 ) and the TNF- $\alpha$ in the plasma of treated rats. CAPE treatment also decreased plasma sICAM-1 levels. This together with decreased inflammatory cellular infiltration into the hepatic tissue as seen during the histological examination may partially contribute to the reduced hepatic necrosis and the decreased production of the inflammatory cytokines. These findings support previous reports about the beneficial effects of CAPE against other types of toxic induced liver failure ${ }^{19,20}$ that was attributed to the very potent antioxidant activity of its major ingredients. Therefore, the antioxidant, anti-inflammatory and NF- $\kappa \mathrm{B}$ inhibitory actions of CAPE could explain our finding of the protective effect of CAPE against LPS/D-GalN induced hepatocellular damage. CAPE treatment significantly reduced the neutrophilc infiltration and intrahepatic hemorrhagic and preserved architecture of the hepatic lobules in treated rats in the current study. This is consistent with previous reports that CAPE can inhibit the caspases 3 and 8 activities by inhibiting the Fas/FasL protein expression ${ }^{19}$ and, can protect against Fas-mediated cell apoptosis. ${ }^{34}$ Neuronal cell damage induced by LPS/GalN in the current study was found to be totally prevented in the CAPE+LPS group which showed preserved astrocytes histological appearance with absent inflammatory cellular infiltration, with no signs of edema or cytoplasmic swelling. The preventive effect of CAPE on the inflammatory cellular infiltration into the hepatic tissue and brain could be directly attributed to its inhibitory effect on NF- $\kappa \mathrm{B}$ activation. Decreased infiltration of the hepatic and brain tissue by inflammatory cells is expected to be a leading step to decreased pro-inflammatory cytokines production and other molecules like, ROS, NO and PGE2 that enhance the pathologic features of inflammation vice vasodilatation and edema. ${ }^{8,13}$

\section{CONCLUSION}

The ability of CAPE to alleviate the SIR, hepatic and neuronal cell damage induced by LPS/D-GalN could be attributed to the antioxidant activity and/or inhibition of free radical generation and the anti-inflammatory action of CAPE. This effect of CAPE was mainly through reversing the imbalance of the pro- and anti-inflammatory cytokines, which may lead to the inhibition of adhesion molecules expression. These results suggest that; the anti-inflammatory action of CAPE, the antioxidant effect, and its ability to inhibit NF- $\mathrm{KB}$ production; are the possible protective mechanisms. CAPE is a promising agent that could help in the prophylaxis and treatment of septic shock.

\section{ACKNOWLEDGEMENTS}

The research team is grateful to the College of Medicine Research Center (CMRC) for supporting this research by the grant number 07-580. We are also appreciative of the technicians in the Physiology and Pathology Departments and the Animal Care Unit in King Khalid University for their expertise and support in accomplishing the practical part of this work.

\section{REFERENCES}

1. Martin GS, Mannino DM, Eaton S, Moss M. The epidemiology of sepsis in the United States from 1979 through 2000. N Engl J Med 2003; 348(16):1546-54.

2. Shapiro N, Howell MD, Bates DW, Angus DC, Ngo L, Talmor D. The association of sepsis syndrome and organ dysfunction with mortality in emergency department patients with suspected infection. Ann Emerg Med 2006; 48(5):583-90.

3. Morikawa A, Sugiyama T, KatoY, Koide N, Jiang GZ, Takahashi $\mathrm{K}$, Tamada Y, Yokochi T. Apoptotic cell death in the response of D-galactosamine-sensitized mice to lipopolysaccharide as an experimental endotoxic shock model. Infect Immun 1996; 64:737-8.

4. Galanos C, Freudenberg MA, Reutter W. Galactosamine-induced sensitization to the lethal effects of endotoxin. Proc Natl Acad Sci USA 1979; 76:5939-43.

5. Decker K, Keppler D. Galactosamine hepatitis: key role of the nucleotide deficiency period in the pathogenesis of cell injury and cell death. Rev Physiol Biochem Pharmacol 1974; 71:77- 106.

6. Pinzani M, Marra F. Cytokine receptors and signaling in hepatic stellate cells. Semin Liver Dis 2001; 21:397-416. 
7. Hanada T, Yoshimura A. Regulation of cytokine signaling and inflammation. Cytokine Growth Factor Rev 2003; 13:413-21.

8. Mitchell JA, Larkin S, Williams TJ. Cyclooxygenase 2 regulation and relevance in inflammation. Biochem Pharmacol 1995; 50:1535-42.

9. Punyadeera C, Schneider EM, Schaffer D et al. A biomarker panel to discriminate between systemic inflammatory response syndrome and sepsis and sepsios severity. J Emerg Trauma Shock 2010; 3(10):26-35.

10. Gutteridge JMC, Mitchell J. Redox imbalance in the critically ill. Br Med Bull 1999;55(1):49-75.

11. Mercer-Jones MA, Heinzelmann M, Peyton JC, Wickel D, Cook M, Cheadle WG. Inhibition of neutrophil migration at the site of infection increases remote organ neutrophil sequestration and injury. Shock 1997; 8(3):193-9.

12. Nathan C. Neutrophils and immunity: challenges and opportunities. Nat Rev Immunol 2006; 6:173-82.

13. Thiemermann C. Nitric oxide and septic shock. Gen Pharmacol 1997; 29:159-66.

14. Nagai T, Inoue R, Inoue H, Suzuki N. Preparations and antioxidant properties of water extract of propolis. Food Chem 2003; 80:29-33.

15. Russo, Long, R, Vanella A. Antioxidant activity of propolis: role of caffeic acid phenethylester and galangin. Fitoterapia 2002; 73(Suppl1):S21-S29.

16. Yildiz Y, Serter M, Ek RO et al. Protective effects of caffeic acid phenethyl ester on intestinal ischemia-reperfusion injury. Dig Dis Sci 2009; 54(4):738-44.

17. Natarajan K, Singh S, Burke Jr. TR, Grunberger D, Aggarwal BB. Caffeic acid phenethyl ester is a potent and specific inhibitor of activation of nuclear transcription factor NF-kappa B. Proc Natl Acad Sci USA 1996; 93:9090-5.

18. Makarov SS, NF- $\kappa B$ as a therapeutic target in chronic inflammation: recent advances. Mol Med Today 2000; 6:441-8.

19. Lee KJ, Choi JH, Khanal T, Hwang YP, Chung YC, Jeong HG. Protective effect of caffeic acid phenethyl ester against carbon tetrachloride-induced hepatotoxicity in mice. Toxicology 2008; 248:18-24.

20. Iraz M, Ozerol E, Gulec $M$ et al. Protective effect of caffeic acid phenethyl ester (CAPE) administration on cisplatin-induced oxidative damage to liver in rat. Cell Biochem Funct 2006; 24(4):357-61.

21. Korish AA. Effect of caffeic acid phenethyl ester on the hemostatic alterations associated with toxic-induced acute liver failure. Blood Coag Fibrinol 2010; 21:158-63.

22. Liu SF, Malik AB, NF- $\kappa B$ activation as a pathological mechanism of septic shock and inflammation. Am J Physiol 2006; 290: L622-L45.
23. Victor VM, Espulgues JV, Hernández-Mijares A, Rocha M. Oxidative stress and mitochondrial dysfunction in sepsis: a potential therapy with mitochondria-targeted antioxidants. Infect Disord Drug Targets 2009; 9(4):376-89.

24. Yachida S, Kokudo Y, Wakabayashi H, Maeba T, Kaneda K, Maeta H. Morphological and functional alterations to sinusoidal endothelial cells in early phase endotoxin-induced liver failure after partial hepatectomy in rats. Virchows Arch 1998; 433:173-81.

25. Secchi A, Ortanderl JM, Schmidt W, Gebhard MM, Martin E, Schmidt H. Effect of endotoximea on hepatic portal and sinusoidal blood flow in rats. J Surg Res 2000; 89:26-30.

26. Pils MC, Pisano F, Fasnacht $\mathrm{N}$ et al. Monocytes/macrophages and/or neutrophils are the target of IL-10 in the LPS endotoxemia model. Eur J Immunol 2010; 40(2):443-8.

27. Doughty L, Clark RS, Kaplan SS, Sasser H, Carcillo J. sFas and sFas ligand and pediatric sepsis-induced multiple organ failure syndrome. Pediatr Res 2002; 52(6):922-7.

28. Essani NA, Fisher MA, Farhood A, Manning AM, Smith CW, Jaeschke H. Cytokine-induced upregulation of hepatic intercellular adhesion molecule-1messenger RNA expression and its role in the pathophysiology of murine endotoxin shock and acute liver failure. Hepatology 1995; 21:1632-9.

29. Antoniades CG, Berry PA, Wendon JA, Vergani D. The importance of immune dysfunction in determining outcome in acute liver failure. J Hepatol 2008; 49(5):845-61.

30. Koksel O, Ozdulger A, Tamer L et al. Effects of caffeic acid phenethyl ester on lipopolysaccharide-induced lung injury in rats Pulm Pharmacol Ther 2006; 19(2):90-5.

31. Henderson Jr. WR, Chi EY, Teo JL, Nguyen C, Kahn M. A small molecule inhibitor of redox-regulated NF kappa B and activator protein-1 transcription blocks allergic airway inflammation in a mouse asthma model. J Immunol 2002; 169:5294-9.

32. Barnes PJ. Nuclear factor-kappa B. Int J Biochem Cell Biol 1997; 29:867-70.

33. Ozyurt H, Ozyurt B, Koca K, Ozgocmen S. Caffeic acid phenethyl ester (CAPE) protects rat skeletal muscle against ischemia-reperfusion-induced oxidative stress. Vascul Pharmacol 2007; 47(2-3):108-12.

34. Choi K, Han YH, Cho C. N-Acetyl cysteine and caffeic acid phenethylester sensitize astrocytoma cells to Fasmediated cell death in a redox-dependent manner. Cancer Lett 2007; 257:79-86. 